\title{
Calidad de vida en pacientes con fibromialgia. Revisión bibliográfica
}

\author{
Quality of Life in Patients with Fibromyalgia: A Literature Review
}

Carla del Olmo Climent, María Cuerda Ballester, David Sancho Cantus

Universidad Católica de Valencia San Vicente Mártir. Valencia, España.

Fecha de recepción: 5 de noviembre de 2018 / Fecha de aceptación: 4 de agosto de 2019

\begin{abstract}
Resumen
Objetivo: Describir el impacto de la fibromialgia sobre el estado de salud y calidad de vida de las personas afectadas por fibromialgia (FM).

Método: Revisión bibliográfica de estudios sobre la calidad de vida relacionada con la salud en personas con fibromialgia, evaluados con instrumentos validados.

Resultados: Se analizaron 31 estudios, los cuales fueron mayoritariamente de diseño observacional transversal. Las dimensiones más afectadas fueron la función física, el dolor y estado mental. Los factores como el sedentarismo y la actividad física fueron significativos en esta población. La prevalencia de fibromialgia fue significativamente más alta en mujeres, casadas, con bajo nivel de estudios y amas de casa.

El instrumento más utilizado para medir la calidad de vida relacionada con la salud fue el cuestionario Fibromyalgia Impact Questionnaire (FIQ).

Conclusiones: Los parámetros estudiados han permitido evaluar las necesidades de los pacientes y alcanzar un conocimiento más integral del proceso de esta enfermedad.
\end{abstract}

Palabras clave: fibromialgia, calidad de vida, dolor, enfermedades reumáticas.

\begin{abstract}
Objective: To describe the impact of fibromyalgia on the state of health and quality of life of people affected by fibromyalgia (FM).

Method: Literature review of studies on the quality of life related to health in people with FM, evaluated with validated instruments.

Results: Thirty-one studies were analyzed, which were mainly of transversal observational design. The most affected dimensions were physical function, pain and mental state. The factors like sedentary lifestyle and physical activity were significant in this population. The prevalence of Fibromyalgia was significantly higher in women, married, with low level of education and housewives.

The most used instrument to measure quality of life related to health was the Fibromyalgia Impact Questionnaire (FIQ).

Conclusions: the parameters studied have allowed to assess the needs of patients and achieve a more comprehensive knowledge of the process of this disease.
\end{abstract}

Keywords: fibromyalgia, quality of life, pain, rheumatic diseases.

\section{Introducción}

La fibromialgia (FM) es la segunda afección más frecuente entre las enfermedades reumáticas. Tiene un curso crónico y un impacto considerable sobre el bienestar físico, psicológico y social del paciente. Está reconocida como un diagnóstico diferenciado desde 1992 por la Organización Mundial de la Salud (OMS) y tipificado en la Clasificación Internacional de enfermedades (CIE-9). Existen controversias sobre su etiología y tratamiento; pese a ello, queda patente la necesidad de enfocar las acciones sanitarias hacia una atención integral de los afectados ${ }^{1}$.

Hoy día, representa uno de los mayores retos planteados a la sanidad, tanto por su elevada prevalencia 
como por las consecuencias que de ella se derivan al paciente, a sus familiares y al entorno sociolaboral ${ }^{2,3}$.

La FM se caracteriza por dolor musculoesquelético generalizado, acompañado de un cuadro sintomático complejo que engloba trastornos del sueño, fatiga intensa, disminución del umbral del dolor y alteración en la dimensión psicológica y afectiva. Este cuadro sintomático conlleva un impacto considerable sobre las actividades cotidianas y la capacidad de trabajo de estos pacientes, repercutiendo en su calidad de vida. Signos y síntomas como rigidez articular, cefalea, vértigo, intestino irritable y síndromes de vejiga, fenómeno de Raynaud, síndrome de piernas inquietas, dismenorrea, sensación de tumefacción en manos, parestesia en extremidades y cambios en el color de la piel reticular, a menudo acompañan a la FM. Igualmente, hipersensibilidad en múltiples puntos predefinidos denominados puntos gatillo o trigger points, los cuales corresponden a zonas fisiológicamente más sensibles a la presión. A pesar del dolor, las estructuras afectadas no presentan daño o inflamación ${ }^{3-5}$.

Una parte importante en la FM son los trastornos psicológicos. En diversos estudios se ha observado que el diagnóstico de episodios depresivos es más frecuente en los pacientes con FM (71\%), comparados con grupos control (12\%). Otros estudios demuestran que la depresión influye en la percepción del dolor musculoesquelético, haciendo que éste se perciba con mayor intensidad y viceversa. Algo similar ocurre con los trastornos de ansiedad, siendo en estos pacientes más frecuentes $(26 \%)$. Aunque la gran mayoría de pacientes no cumple criterios de trastorno psiquiátrico, los síntomas psicológicos son comunes. Se considera la segunda patología reumática más frecuente, superada por la osteoartritis y tiene una prevalencia poblacional que varía entre el $10 \%$ y $15 \%$, según un estudio realizado en diversos países europeos ${ }^{6,7}$.

En el ámbito nacional, la prevalencia es del 0.7-10\% de la población. El estudio EPISER 2000 es uno de los proyectos estratégicos de la Sociedad Española de Reumatología (SER), así como el estudio de la prevalencia de las enfermedades reumáticas en la población española adulta mayor de 20 años. Este fue el primer estudio de base poblacional representativo en población adulta de España, en el que se estimó la prevalencia e impacto de 6 entidades reumáticas, entre ellas la FM. Sus resultados se consideran representativos de la población española ${ }^{8}$.
Centrándonos en la sintomatología, los resultados muestran que el $8.1 \%$ de las personas encuestadas refería dolor musculoesquelético generalizado el día de la entrevista y un $5.1 \%$ del total cumplía requisitos para considerarlo crónico (duración superior a 3 meses). La mayor parte de estos pacientes presentaban dolor a la palpación en varias zonas del cuerpo. En la población mayor de 20 años, esto sucedía en el $2.4 \%$, lo que representa en valores absolutos que en España hay unas 700000 personas afectadas por la FM y cada año se diagnostican unos 120000 casos. Si nos fijamos en la diferencia entre sexos, se observa que en hombres se estima en un $0.2 \%$, frente a un $4.2 \%$ en las mujeres, lo que supone una relación de prevalencia hombremujer de $1: 21^{8,9}$.

Durante el año 2016, la SER propuso la realización de un nuevo estudio epidemiológico de enfermedades reumáticas en adultos, dado los importantes cambios poblacionales que se han producido en nuestro país y que podrían influir en la prevalencia de las enfermedades reumáticas. En colación a esta propuesta y según datos del Instituto Nacional de Estadística, se ha observado que las personas mayores de 64 años han aumentado en un $2.2 \%$ el índice de envejecimiento, el aumento del porcentaje de la población extranjera ha sido de un $5.3 \%$ y el sobrepeso y la obesidad se han incrementado en un $17.6 \%$ y un $3.6 \%$ respectivamente (datos de la Encuesta Nacional de Salud de 2000 y la Encuesta Europea de Salud de 2014). Todos estos cambios en factores que influyen en las enfermedades reumáticas hacen que esté más que justificada la reedición del estudio EPISER ${ }^{10,11}$.

Por edades, la FM se manifiesta en todos los grupos de edad, aunque existe una prevalencia máxima entre los 40 y los 49 años siendo escasamente frecuente en mayores de 80 años. No obstante, la diversidad sintomatológica del cuadro de FM y las dificultades para llegar a un diagnóstico preciso afectan directamente al consenso sobre su prevalencia, que difiere según los estudios, barajándose cifras de un $2-4 \%$ en la población general, entre un 2-6\% en las consultas de atención primaria, llegando hasta un 10-20\% en las consultas de reumatología $a^{3,12}$.

\section{Calidad de vida en pacientes con fibromialgia}

En este apartado se mezclan dos términos, la calidad de vida en relación a la salud (CVRS) y la calidad de vida (CV). 
Por una parte, la CVRS hace mayor énfasis en la evaluación, por parte del individuo, de las limitaciones en las áreas biológicas, psicológicas y sociales que ha podido desarrollar la paciente a partir de su enfermedad. Este concepto es importante, ya que el bienestar de los pacientes es un punto crucial para considerar el tratamiento adecuado sin mermar su bienestar ${ }^{13}$.

Algunos autores consideran la CVRS como una parte de la CV general, mientras que otros sugieren que ambos constructos estarían evaluando similares dimensiones. Pese a esta discrepancia, la gran mayoría de los autores sugiere que la CV debe ser diferenciada de la CVRS debido a que el término es utilizado en el campo de la medicina en su interés por evaluar la calidad de los cambios, como resultado de intervenciones médicas. Así pues, debe limitarse para medir la experiencia que el paciente tiene de su enfermedad, así como el resultado obtenido de los cuidados médicos o para establecer el impacto de la enfermedad en la vida diaria. A consecuencia de ello, lo consideramos como una medición de salud desde la perspectiva de los pacientes $^{14}$.

Instrumentos de valoración más utilizados en la evaluación de la fibromialgia

Los instrumentos o escalas de medida de la CVRS incluyen diferentes ítems que permiten la evaluación del funcionamiento físico, social y emocional. En el ámbito general, se pueden aplicar en un amplio espectro de enfermedades y en toda la población. En el ámbito específico, se centran en aspectos más concretos de la salud y en una sola patología determinada. Estos instrumentos deben tener propiedades que nos garanticen la validez (veracidad de la prueba) y la fiabilidad (consistencia de la prueba). Un estudio reciente encontró que los instrumentos más utilizados para medir la CVRS en personas con FM fueron las escalas de ámbito general: Medical Outcome Study Short Form (MOSSF-36) y World Health Organization Quality Of Life (WHOQOL-BREF) y en el ámbito específico: Fibromyalgia Impact Questionnaire 1994 (FIQ), los cuales han sido adaptados a diferentes países e idiomas y presentan una buena estructura conceptual, excelente validez y fiabilidad $^{1,15}$.

Por una parte, el cuestionario FIQ evalúa el impacto de la FM en 10 dimensiones: capacidad física, trabajo habitual, afectación de la actividad laboral remunerada, dolor, fatiga, sensación de cansancio, rigidez, sensación de bienestar, ansiedad y depresión. En otras palabras, evalúa los síntomas psicológicos y físicos, lo que permite una medición amplia de los diferentes indicadores de FM.

Por otro lado, el cuestionario MOSSF-36, permite evaluar el estado de salud y CVRS en personas sanas y enfermas. Genera un perfil de 8 dimensiones: dolor corporal, desempeño físico, función física, salud general, desempeño emocional, función social, salud mental y vitalidad ${ }^{1,15}$.

Las intervenciones de enfermería en pacientes con FM son esencialmente de carácter paliativo y educativo; se llevan a cabo principalmente en escenarios comunitarios, con el propósito de aprender a manejar el dolor, disminuir la ansiedad, mejorar el patrón de sueño y conservar la energía para la realización de las actividades básicas e instrumentales de la vida cotidia$\mathrm{na}^{16}$.

Basándonos en la información recabada, nuestro objetivo principal es conocer mediante una revisión bibliográfica de la literatura científica el impacto de la fibromialgia sobre el estado de salud y calidad de vida de las personas afectadas.

\section{Material y métodos}

Revisión bibliográfica de la literatura sobre la CVRS en pacientes con FM. Las variables incluidas en el estudio fueron la función física, salud mental, función social, función cognoscitiva, sueño y descanso, alimentación, función del rol, fatiga, función sexual, dolor, salud percibida, impacto de la enfermedad y variables sociodemográficas. Aunque las más comúnmente evaluadas fueron el dolor, la calidad de vida y la funcionalidad.

La búsqueda se realizó entre los meses de diciembre de 2017 y mayo de 2018. Las bases de datos que se emplearon para hacer la búsqueda de la literatura científica fueron Cuiden, Pubmed, Scielo, Redalcy y Dialnet.

Los descriptores utilizados fueron "Fibromialgia", "Fibromyalgia", "Calidad de vida", "Quality of life", "Calidad de vida relacionada con la salud", "Health related quality of life".

Se siguieron una serie de criterios para la elección de los distintos artículos, los cuales se especifican a continuación: 


\section{Criterios de inclusión:}

- Estudios en castellano e inglés.

- Estudios en los que se hayan empleado instrumentos de medición de la CVRS validados por expertos.

- Estudios con consentimiento informado de los pacientes.

- Artículos publicados durante los últimos 6 años.

- Pacientes adultos.

- Pacientes diagnosticados según el criterio de clasificación del Colegio Americano de Reumatología para pacientes con FM.

\section{Criterios de exclusión:}

- Estudios que aplicaban las escalas de valoración a la población juvenil.

- Estudios sin texto completo.

- Estudios que no evaluaban la CVRS en pacientes con FM.

- Estudios que no son originales.

- Estudios que no incluyesen escalas de valoración de CVRS.

\section{Selección de artículos}

La búsqueda de la información se realizó siguiendo el protocolo DETAC de investigación, que tiene como objetivo minimizar errores, ayudar a la planificación y realización de estudios de adaptación transcultural y validación de los cuestionarios sobre calidad de vida. El protocolo consta de 5 pasos, y cada uno de ellos se refiere a una fase de investigación: $\mathrm{D}$ : definir; $\mathrm{E}$ : esquematizar; T: traducir; A: aplicar; C: consolidar, analizar y presentar los datos del estudio. Siguiendo los pasos se consideró en cada estudio los objetivos, los problemas planteados, los instrumentos para evaluar la validez de contenido, la reproducibilidad y la coherencia interna, además de si valoraba la respuesta a la intervención y si la muestra de población era representativa de la comunidad estudiada.

Se seleccionaron todos aquellos artículos que incluían escalas de valoración de la CVRS en pacientes con FM, mediante cuestionarios genéricos u específicos de la enfermedad. No obstante, se priorizaron aquellos estudios que utilizaron cuestionarios multidisciplinares específicos, ya que se aprovechó para examinar otras dimensiones más allá de los síntomas físicos como, por ejemplo, el impacto social, emocional y otras actividades que impactan en la vida diaria.

\section{Resultados}

El diseño de estudio más utilizado fue el de tipo observacional transversal, presente en 16 de los 31 artículos elegidos. En cuanto al idioma, 16 fueron publicados en inglés y los restantes en español. El tamaño muestral medio de los estudios fue de 921.61 sujetos, aunque se distingue una gran variabilidad de estas cifras en cada estudio, con muestras que van desde los 27 pacientes a un máximo de 6394 participantes.

\section{Características de la población}

En cuanto a las características de los individuos estudiados, eran personas con diagnóstico clínico reumatológico de FM según el American College of Rheumatology (ACR) y la edad promedio de los seleccionados fue de 48 años. Por otra parte, la duración media de la enfermedad fue de 7.52 años, la de los síntomas que presentaban los individuos fue de 9.5 años y el promedio de años desde el diagnóstico fue de 6.8 años.

En 11 de los 31 artículos seleccionados, la población estudiada fue exclusivamente femenina, 17 artículos incluyeron personas de ambos sexos y los estudios restantes no especificaron dicho dato. En todos los estudios que incluían tanto población masculina como femenina, la muestra de mujeres fue notablemente superior a la de hombres, probablemente justificado por la alta prevalencia de la enfermedad en la población femenina.

Por lo que respecta a la procedencia de los pacientes, encontramos que, en 20 artículos, los pacientes fueron cribados a través de las referencias de los servicios de reumatología de los centros hospitalarios y centros ambulatorios, mientras que fueron 6 los artículos que reclutaron la población a través de asociaciones de pacientes con FM y 5 artículos analizaron la población de otros ensayos ya existentes.

Los estudios con datos sociodemográficos disponibles describen que la mayoría de los individuos eran mujeres de media edad, casadas, amas de casa la mayoría y una minoría de mujeres activas. El nivel de estudios predominante entre los individuos era educación secundaria, encontrando sólo dos estudios que el nivel educativo era universitario. El nivel económico de la población estudiada fue por lo general medio. Centrando la atención en el consumo de tabaco y alcohol, los estudios revelaron que los individuos no eran usuarios de estas sustancias, sin embargo, muchos de ellos sí 
tomaban antidepresivos, benzodiacepinas o antinflamatorios. En cuanto a la presencia de sedentarismo y la falta de actividad física, fue significativa en esta población, hecho vinculante al sobrepeso que presentaba la mayoría de los pacientes y algunos artículos señalaron la presencia de otras comorbilidades simultáneas con la FM.

El instrumento más utilizado para medir la CVRS fue el del ámbito específico, FIQ, concretamente en 17 artículos, seguido en segundo lugar por el cuestionario de ámbito genérico MOSSF-36, el cual utilizaron 15 artículos.

La mayoría de estudios evaluaron el estado mental de los pacientes antes de incluirlos en la investigación, ya que algunos artículos presentaban como criterio de exclusión la presencia de algún tipo de enfermedad mental. Otros consideraron el factor psicológico en su estudio como importante dada la gran influencia que tiene en los pacientes con FM. Para ello, las escalas más utilizadas fueron HADS (Hospital Anxiety and Depression Scale), seguida de la STAI (State-Trait Anxiety Inventory) y BDI (Beck Depression Inventory). Casi la totalidad de los estudios que evaluaron esta dimensión evidenciaron sintomatología ansiosa, depresiva o ambas en los individuos del estudio, lo cual tenía un impacto importante en la salud percibida y $\mathrm{CV}$ de las personas con esta enfermedad.

Otras áreas afectadas y que presentaron mayor impacto fueron la función física, la salud general y el dolor. También se observaron alteraciones en el patrón sueño-descanso.

\section{Discusión y conclusiones}

Todos los estudios coincidieron en que en los grupos etarios afectados por la FM existe mayor frecuencia de sexo femenino en razones mayores a 7:1. Aunque el estado de salud y el perfil de calidad de vida no presentaron asociación estadística con el sexo, podemos observar que esta enfermedad tiene mayores impactos negativos sobre las mujeres, ya que, generalmente, tiene mayores exigencias y responsabilidades ${ }^{17}$.

Los estudios de Miró et al. ${ }^{18}$ y Segura et al. ${ }^{19}$ analizaron si las manifestaciones de la FM diferían en función del sexo. Encontraron que la diferencia más importante entre sexos fue que las mujeres presentaban un menor umbral de sensibilidad al dolor en el algiómetro e informaban de un mayor consumo de analgésicos. El aspecto físico del dolor crónico en FM puede conducir al desarrollo de diversos problemas de salud mental, repercutiendo así en la CV de las personas con FM. Por tanto, se puede observar que existe una estrecha relación entre las variables físicas y psicológicas y el gran impacto que tienen en esta enfermedad.

Siguiendo con estas diferencias entre géneros y la menor prevalencia de la enfermedad en hombres, Segura et al. ${ }^{19}$ demostraron que el punto de corte para el diagnóstico de FM en los hombres debe ser mayor que el de las mujeres.

Muchos estudios coinciden en sus reflexiones al evaluar la FM desde un aspecto biopsicosocial, ya que es una manera holística multidimensional de captar los efectos que produce la enfermedad en su vida cotidiana, mediante la intervención de un equipo multidisciplinar, que permita formular un diagnóstico y tratamiento adecuado para cada paciente y mejorar así su CV.

Cardona-Arias et al. ${ }^{17}$ en su estudio observaron que las dimensiones con mayor deterioro fueron las del componente físico: la actividad física, la participación social o comunitaria, la escolaridad y la edad. Estos resultados fueron similares a otros estudios en los que se compararon las dimensiones de $\mathrm{CV}$ en personas con FM frente a otras enfermedades reumatológicas y frente a personas sanas, lo que evidencia su peor estado de salud y de calidad de vida ${ }^{4,13,18-25}$.

Los dos cuestionarios más utilizados en los estudios analizados fueron el SF-36 y el FIQ; de hecho, muchos estudios aprovecharon para remarcar la fiabilidad y validez de estos cuestionarios e indicaron la importancia de su utilización en la medición de la CVRS ${ }^{4}$.

Las dimensiones más afectadas en todos los casos fueron las correspondientes a los "problemas físicos" seguida por la del "dolor" 26 . Analizando las escalas de forma separada, las más afectadas en el SF-36 fueron la función física, el dolor, salud general y salud mental, mientras que las del FIQ fueron dolor, fatiga, ansiedad, depresión y el bienestar ${ }^{20,24,25}$.

No debemos olvidar que el dolor crónico es el principal problema de estos pacientes; ellos lo describen como incapacitante para la actividad de la vida diaria pero no para la movilidad. Cabe añadir que es resistente a los diagnósticos y a los tratamientos convencionales ${ }^{3}$. 
Los valores de ansiedad y depresión reflejados en los estudios analizados fueron altos, tal y como se muestra en el estudio de Alvarado Moreno et al. $^{7}$; estas alteraciones derivan en deterioros en el resto de las esferas.

Desde una perspectiva biopsicosocial, el aspecto físico del dolor crónico puede conducir al desarrollo de una variedad de problemas de salud mental; por lo tanto, podemos pensar que el dolor se vuelve central y los pacientes se ven atrapados por é $1^{4,21,26}$.

Keller et $a l .{ }^{12}$ destacan que la heterogeneidad de la FM se debe principalmente a diferencias en la sintomatología ansiosa, depresiva y una afectación de la esfera psicopatológica, lo que puede indicar una comorbilidad psiquiátrica en los pacientes con FM tipo I, es decir, aquella en la que los pacientes no tienen enfermedades concomitantes. Esta comorbilidad suele ser frecuente en enfermedades crónicas y suele presentarse como una forma de depresión enmascarada.

Otra de las dimensiones afectadas por la FM y no menos importante es el sueño. Los resultados de Wagner et al..$^{27}$ sugirieron que más del $88 \%$ de los pacientes reportaron dificultades para dormir, al conciliar el sueño, a permanecer dormido o a despertarse temprano. Esto se apoya con el estudio de Liedberd et al. $^{28}$, donde el grupo de pacientes con mal sueño obtuvieron peores puntuaciones al dolor, factores psicológicos, alteración de la funcionalidad y $\mathrm{CV}$ percibida.

La fatiga fue manifestada por muchos pacientes como otro de los peores síntomas asociados con la FM. Algunos pacientes también manifestaron cierto deterioro cognitivo, en memoria y pensamiento, que se habían visto afectados desde la aparición de la enfermedad.

Elizagaray et $a l .{ }^{29}$ incide en la importancia que tiene la educación al paciente, ya que se mostró evidencia moderada-fuerte de sus efectos beneficiosos sobre el dolor, funcionalidad y $\mathrm{CV}$ a corto, medio y largo plazo al combinarse con ejercicio y una intervención de afrontamiento activo.

La CVRS en los pacientes con FM resulta de gran relevancia para complementar indicadores tradicionales de morbilidad, mortalidad y expectativa de vida, orientar la toma de decisiones sobre tratamientos médicos, establecer prioridades en salud y diseñar, imple- mentar, seguir y evaluar programas de prevención, atención y rehabilitación ${ }^{20,21,29-33}$.

Dentro de las limitaciones que podemos encontrar en este estudio, hay que señalar que la muestra ha considerado un número limitado de artículos; no obstante, los hallazgos han permitido elaborar una reflexión profunda del tema.

Otra de las limitaciones es que muchos estudios no consideraron a los hombres y, en los pocos que sí lo hacen, la muestra fue significativamente menor a la de las mujeres.

En líneas de investigación futuras, proponemos realizar estudios experimentales para conseguir una aproximación más real al modo en que conciben la calidad de vida los pacientes con FM.

Por todo lo expuesto, podemos concluir que las personas con fibromialgia manifiestan una falta de comprensión y aceptación social. No hay evidencia de enfermedad porque se desconoce su causa y no hay ninguna prueba médica que pueda corroborarla. Esa incertidumbre lleva a un punto de inflexión: la necesidad de un diagnóstico firme que disminuirá la ansiedad y desarrollará estrategias de afrontamiento en el paciente en este proceso de salud-enfermedad.

Se ha descrito la importancia de usar escalas genéricas y específicas de forma combinada para la evaluación de la calidad de vida relacionada con la salud en fibromialgia.

Una comprensión profunda de la experiencia de la enfermedad es clave en el cuidado de estos pacientes; el conocimiento de la realidad y el significado de vivir día a día con esta enfermedad debería ser considerado esencial para cualquier profesional sanitario incluyendo la necesidad de un proceso diagnóstico eficaz y una mayor educación acerca de la experiencia de la fibromialgia.

Nos gustaría señalar la importancia de abrir nuevas vías de ayuda y centrar la importancia para que todos los profesionales puedan conocer más la enfermedad, y así poder prestar a los pacientes la atención, orientación y los cuidados necesarios con el fin de ayudarlos en el proceso degenerativo de la enfermedad, mejorar su calidad y esperanza de vida. 
Finalmente, queremos resaltar la importancia de crear consciencia a la población sobre la existencia de esta enfermedad y de sus consecuencias, para que se apoye a su investigación y poder lograr un avance para su mejoría.

\section{Bibliografía}

1. Hernández- Petro AM, Cardona Arias A. Efecto de la Fibromialgia sobre el estado de salud y la calidad de vida relacionada con la salud, 2004-2014Revista Colombiana de Reumatología. 2015; 22(2): 110-118.

2. Pereira de Melo F, de Paiva JA, de Almeida E, Pereira E, Marques M. Relationship between health-related quality of life and sexuality in women with fibromyalgia. Journal of Nursing UFPE On Line. 2014; 8(10): 3587-3593.

3. Triviño Martínez Á, Solano Ruiz MC, Siles González J. La cronicidad de la fibromialgia. Una revisión de la literatura. Enfermería Global. 2014; 13(35):273-292.

4. Sañudo JI, Corrales Sánchez R, Sañudo B. Nivel de actividad física, calidad de vida y niveles de depresión en mujeres mayores con fibromialgia. Escritos de Psicología. 2013; 6(2):53-60.

5. Kucukali Turkyilmaz A, Eda Kurt E, Karkucak M, Capkin E. Sociodeographic Characteristics, Clinical Signs and Quality of Life in Patients with Fibromyalgia. The Eurasian Journal of Medicine. 2012; 44(2):88-93.

6. Latorre Roman Á, Santos e Campos MA, Mejía Meza JA, Delgado Fernández M, Heredia JM. Analysis of the physical capacity of women with fibromyalgia according to the severity level of the disease. Revista Brasileira de Medicina do Esporte. 2012; 18(5): 308-312.

7. Alvarado Moreno JG, Montenegro Díaz KA, León Jiménez FE, Díaz Vélez C. Asociación entre Depresión-Ansiedad y el Síndrome de Fibromialgia en 3 centros asistenciales de Lambayeque, Perú, 2011-2012. Revista Médica Risaralda. 2014; 20(2): 75-79.

8. Bustabad S, Díaz González F. Estudio EPISER 2016. el porqué de un nuevo estudio epidemiológico. Reumatología Clínica. 2017; 13(1):1-3.

9. Collado Cruz A, Cuevas Cuerda MD, Estrada Sabadell MD, al. e. Ministerio de Sanidad, Servicios Sociales e Igualdad. [Online]; 2011 [cited 2017 febrero. Available from:

https://dialnet.unirioja.es/servlet/articulo?codigo $=5783678$

10. Estadística INd. INE. [Online].; 2014 [cited 2018 Noviembre 3. Available from:

http://www.ine.es/en/metodologia/t15/t153042014_en.pdf

11. Pública INdS. INSP. [Online].; 2000 [cited 2018 Noviembre 3. Available from: https://www.insp.mx/encuestoteca/Encuestas/ENSA 2000/OTROS/ensa_tomo2.pdf

12. Keller D, de Gracia M, Cladellas R. Subtipos de pacientes con fibromialgia, características psicopatológicas y calidad de vida. Actas Españolas de Psiquiatría. 2011;39(5):273-279.

13. Cardonas Arias JA, Hernández Petro M, León Mira V. Validez, fiabilidad y consistencia interna de tres instrumentos de medición de calidad de vida relacionada con la salud en personas con fibromialgia, Colombia. Revista Colombiana de Reumatología. 2014; 21(2): 57-64

14. Urzúa M A. Calidad de vida relacionada con la salud: Elementos conceptuales. Revista Médica de Chile. 2010 marzo; 138(3): 358365.

15. Lemus N, Parrado R, Quintana G. Calidad de vida en el sistema de salud. Revista Colombiana de Reumatología. 2014; 21(1):1-3.

16. Restrepo Medrano JC, Rojas JG. Fibromialgia ¿Qué deben saber y evaluar los profesionales de enfermería? Investigación y Educación en Enfermería. 2011;29(2):305-314.

17. Cardona Arias JA, León Mira V, Cardona Tapias AA. Estado de salud y calidad de vida en pacientes con fibromialgia, Medellín. Revista
Colombiana de Reumatología. 2014; 21(1):10-20.

18. Miró E, Diener FN, Martínez MP, Sánchez Al, Valenza MC. La fibromialgia en hombres y. mujeres: comparación de los principales síntomas clínicos. Psicothema. 2012;24(1):10-15.

19. Segura Jiménez V, Estévez López F, Soriano Maldonado A, al. e. Gender Differences in Symptoms, Health-Related Quality of Life, Sleep Quality, Mental Health, Cognitive Performance, PainCognition, and Positive Health in Spanish Fibromyalgia Individuals: The Al-Ándalus Project. Pain Research and Management. 2016; 2016: 1-14.

20. C Branco J, M Rodrigues A, Gouveia N, Eusébio M, Ramiro S, M Machado $P$, et al. Prevalence of rheumatic and musculoskeletal diseases and their impact on health-related quality of life, physical function and mental health in Portugal: results from EpiReumaPt- a national health survey. Rheumatic and Musculoskeletal Diseases. 2016; 2:2-12.

21. Lee JW, Lee KE, Park DJ, Kim SH, Nah SS, Ji Hyun Lee ea. Determinants of quality of life in patients with fibromyalgia: A structural equation modeling approach. PLoS ONE. 2017;12(2):1-12.

22. A Aparicio V, B Ortega F, Carbonell Baeza A, Camiletti D, R Ruiz J, Delgado Fernández M. Relationship of Weight Status with Mental and Physical Health in Female Fibromyalgia Patients. Obesity Facts. The European Journal of Obesity. 2011;4(6):443-448.

23. Schaefer C, Chandran A, Hufstader M, Baik R, McNett M, Goldenberg $D$, et al. The comparative burden of mild, moderate and severe Fibromyalgia: results from a cross- sectional survey in the United States. Health and Quality of Life Outcomes. 2011; 9:71.

24. García HI, Vera Cl, Lugo LH. Calidad de vida relacionada con la salud en Medellín y su área metropolitana, con aplicación del SF-36. Revista de la Facultad Nacional de Salud Pública.2013; 32(1): 2639.

25. Del Río González E, García Palacios A, Botella C. Calidad de vida en fibromialgia: influencia de factores físicos y psicológicos. Psicología Conductual. 2014;22(1):19-35.

26. Martín Nogueras AM, de Sousa Pinto JM, Calvo Arenillas JI. Evaluación de la calidad de vida relacionada con la salud en pacientes con fibromialgia. Revista Brasileira en Promoçao da Saúde. 2010; 23(3): 99-205.

27. Wagner JS, DiBonaventura MD, Chandran AB, Cappelleri JC. The association of sleep difficulties with health-related quality of life among patients with fibromyalgia. BMC Musculoskeletal Disorders. 2012; 13: 1-10.

28. Kim SK, Kim SH, Lee CK, Lee Hs, Lee SH, Park YB, et al. Effect of fibromyalgia syndrome on the health-related quality of life and economic burden in Korea. Rheumatology (Oxford).2013; 52(2): 311-320.

29. Elizagaray García i, Muriente González J, Gil Martínez A. Educación al paciente con fibromialgia. Revisión sistemática de ensayos clínicos aleatorizados. Revista de Neurología. 2016; 62: 49-60.

30. Cardona Arias A, León Mira V, Cardona Tapias AA. Calidad de vida relacionada con la salud en adultos con fibromialgia, 2012. Revista Colombiana de Reumatología. 2013;20(1): 19-29.

31. El Miedany Y, El Gaafary M, Youssef S, Ahmed I. Towards Tailored Patient's Management. Approach: Integrating the Modified 2010 ACR Criteria for Fibromyalgia in Multidimensional patient Reported Outcome Measures Questionnaire. Hindawi. 2016;2016: 9-16.

32. Danet A, Prieto Rodríguez MÁ, Valcárcel Cabrera MC, March Cerdà JC. Evaluación de una estrategia formativa entre iguales en fibromialgia. Un análisis de género. 2016; 16(3):296-312.

33. Salaffi F, Sarzi Puttini P, Girolimetti R, Atzeni F, Gasparini S, Grassi W. Health-related quality of life in fibromyalgia patients: a comparison with rheumatoid arthritis patients ans the general population using the SF-36 health survey. Clinical and Experimental Rheumatology. 2009; 27(56): 67-74 